\title{
Patient Expectation of a Voice Clinic Consultation: Development of the ACaPELa Questionnaire by Assessment of Four Hundred and Fifty Five Patients.
}

Mr Robin Crosbie, ENT Department, Glasgow Royal Infirmary

Dr Mel McKendrick, Humanities and Social Sciences Department, University of Strathclyde, Glasgow Dr Stephen Corson, Mathematics and Statistics Department, University of Strathclyde, Glasgow Dr Anja Lowit, Speech and Language Department, University of Strathclyde, Glasgow Professor Kenneth MacKenzie, ENT Department, Glasgow Royal Infirmary, Glasgow

Corresponding author:

Mr Robin Crosbie, ENT Department, Glasgow Royal Infirmary, Castle Street, Glasgow.

Email address: crosbie.robin@gmail.com 


\section{Key Points}

1. We need to understand our patients' expectations to be able to improve subjective outcomes, whilst delivering the best evidence-based care possible.

2. We believe we are the first to present data reporting patients' expectations in a Voice Clinic patient cohort.

3. There is a lack of evidence and understanding of patient expectation and its relationship with patient care.

4. It is envisaged that our ACaPELa questionnaire will be used in routine clinical practice to not only establish patients' expectation from a laryngology consultation, both clinical and therapy based, but will also be used post consultation to assess whether these expectations have been achieved, or not. 


\section{Introduction}

The many forms of dysphonia present a significant management challenge. Not only may it have a negative effect on a patients' quality of life ${ }^{1}$, but in a proportion of patients it may be the presenting symptom of a head and neck malignancy. As a result, General Practitioners (GPs) are advised to refer any patient with persistent dysphonia for longer than three weeks for ORL assessment ${ }^{2,3}$.

Although patients presenting with dysphonia may have been referred by their GPs to exclude a malignancy, patients themselves may have multiple expectations ranging from exclusion of serious disease to complete resolution of every facet of their vocal issues. Equally, ORL clinicians may pursue management on what they perceive are the most relevant clinical issues. Given these uncertainties there is likely to be a mismatch of referral, expectation and subsequent management.

If it is possible to identify the patients' expectations of the outpatient consultation then it may be possible to not only tailor their consultation more effectively but also ensure that their management is optimised. The aim of the study was to assess patients' expectations of the consultation at a specialist voice clinic and identify a series of questions which would reflect these expectations.

\section{Patients and Methods}

All patients attending the adult Voice Clinic at Glasgow Royal Infirmary between January 2012 and July 2014 were included. A total of 521 patients were asked to participate in the study, 71 patients had to be excluded due to incomplete datasets, resulting in 455 for analysis. The mean age at the clinic visit was 52.3 years (SD 18.8, range 14-100 years). There were 288 females and 167 males in the study population (ratio 1.7:1). All participants were invited to complete two questionnaires prior to their outpatient consultation. The first was a specifically designed questionnaire which included fourteen questions assessing patient expectations (PEQ) from the clinic, with a final question asking for 'anything else' (Figure 1). The fourteen questions had been derived from a previous pilot study, where a similar group of patients had been given an open structure questionnaire and were asked to list all the questions they wished addressed from the consultation. To determine the degree of dysphonia, patients were asked to complete the Voice Symptom Score $(\text { VoiSS })^{4}$, which is a validated self-assessment questionnaire of vocal quality. 
Each patient was assessed in the Voice Clinic by our lead author (KMK), thus reducing interobserver variability, with the diagnosis and subsequent management recorded. The anonymised data were analysed using password-protected NHS computers. The project was accepted by the local Clinical Effectiveness Department as a clinical audit, therefore did not require specific ethical approval.

\section{Results}

The most common diagnoses were functional dysphonia and laryngitis. Other conditions included; chronic rhinitis, neck lumps, tonsillar pathology, subglottic stenosis, connective tissue disorders, neurological disease or pharyngeal pouch. Of note, there were $21(4.6 \%)$ cases of possible head and neck malignancy in the study group; 5 (1.1\%) of which were subsequently confirmed histologically.

The mean total VoiSS score was 36.86 (SD 21.63, median 33, range 0-106); mean impairment subscale was 21.61 (SD 13.06, median 20, range 0-57), mean emotion subscale was 6.44 (SD 7.56, median 4, range 0-32) and mean physical subscale was 9.02 (SD 4.9, median 9, range 0-25). This compares to a 'normal' population, where the total VoiSS score is between 0 and 12.

The PEQ results, shown in Table 1, demonstrate that the most common expectations were for Question 13 (GP/other doctor wanted me checked out), Question 2 (I want to know what is wrong with my throat), Question 3 (I want to know what is wrong/ I want a diagnosis), Question 1 (I want to know what is wrong with my voice) and Question 9 (Tell me what I can do to make my throat problem better). Of particular interest in the other expectations, we found that $49.7 \%$ of patients reported wanting to know if they have cancer (Question 4); although only 44\% of the patient cohort were referred on the "urgent suspicion of cancer" pathway. There were 19 responses (4.2\%) to Question 15 (Anything else); all were covered by the other questions or related to nonlaryngological symptoms the patients were experiencing. The mean number of responses to questions for the PEQ was 7.6 (SD 3.3, range 0-14). Internal consistency of the questionnaire was high with Cronbach's Alpha of 0.81 .

There was no significant variation in the mean age of the patients answering each question within the PEQ; shown in Table 2. There were however a slightly higher mean age of patients wanting to know if they had cancer, find out why they had lots of phlegm in their throats and those who did not 
know why they were at the clinic; 55.7, 55 and 58.4 years respectively. Equally, there was a slightly lower mean age of patients wanting their singing voice improved (48.5 years).

Pearson's correlation revealed a significant but weak positive correlation between the PEQ as a whole (all questions) with each of the VoiSS subscales along with the total VoiSS scores. Impairment scale (I) $(r=0.32, p<0.001$, one-tailed $)$ explaining only $10 \%$ of the variance; emotional scale (E) $(r=0.25, p<0.001$, one-tailed) with only $6 \%$ of the variance; physical scale (P) ( $r=0.26, p<0.001$, one-tailed); total score $(r=0.36, p<0.001$, one-tailed $)$ with $13 \%$ of the variance.

To improve the internal reliability of the full questionnaire (Figure 1), Cronbach's alpha was used to identify dispensable variables - that is variables which, if removed, would result in an increase in the baseline Cronbach's alpha score. The systematic process indicated that removal of six questions (Questions 6-8 and Questions 12-14) would increase the internal reliability of the questionnaire from 0.81 to 0.83 . The subsequent questionnaire was termed "Assessing and Caring for Patient Expectations in Laryngology" (ACaPELa) shown in Figure 2.

\section{Discussion}

Managing patients' expectations can be challenging. It has been suggested by psychologists that the difference between what is expected to be received and what is received, determines overall satisfaction $^{5}$. This is important within the Healthcare service, as it highlights the link between the preconceived ideas a patient has for the clinical encounter, how they are managed and their subsequent degree of satisfaction.

Assessing patient satisfaction, or subjective outcomes, in ENT has been extensively developed during recent years, in particular for dysphonic patients; the widespread use of the Voice Handicap Index (VHI) ${ }^{6}$, Voice Handicap Index-10 (VHI-10) ${ }^{7}$ and Voice-Related Quality of Life (V-RQOL) ${ }^{8}$ tools in assessing disease-specific quality of life is supporting evidence. Equally, the development and increasing use of the Glasgow Benefit Inventory (GBI) tool, as a post-intervention assessment of patient satisfaction, is a good example ${ }^{9}$. To develop this phenomenon further we need to understand our patients' expectations to be able to improve subjective outcomes, whilst delivering the best evidence-based care possible. 
We believe we are the first to present data reporting patients' expectations in a Voice Clinic patient cohort. There have been other studies recently in ENT exploring patient expectations in relation to day-surgery experience and general outpatient service ${ }^{10,11}$. These papers highlight the lack of evidence and understanding of patient expectation and its relationship with patient care.

The open-structure design of the pre-study initial pilot questionnaire allowed the creation of the PEQ incorporating a variety of expectations, which covered all the aspects our patient group were keen to explore during the clinic visit, with the subsequent 14 questions being introduced to a large Glasgow patient cohort. A potential limitation of the study is that the PEQ, and subsequent ACaPELa questionnaire, have been derived using expectations of a patient cohort within Glasgow, and therefore may not represent other general populations' expectations. We are of the opinion, however, that the questions are presented in a general form, so that application should not be hindered by geography or other patient factors. To confirm applicability, we would aim to use the ACaPELa questionnaire in a further large audit of clinical practice within our own region, and ideally with colleagues throughout other countries.

It is envisaged that ACaPELa will be used in routine clinical practice to not only establish patients' expectation from a laryngology consultation, both clinical and therapy based, but will also be used post consultation to assess whether these expectations have been achieved, or not.

\section{Conflicts of Interest}

None declared. 


\section{References}

1. Merrill R.M., Roy N., Lowe J. (2013) Voice-related symptoms and their effects on quality of life. Ann. Otol. Rhinol. Laryngol. 122, 404-11.

2. National Institute for Health and Care Excellence (2005). Referral guidelines for suspected cancer; available at http://www.nice.org.uk

3. Scottish Intercollegiate Guidelines Network (2006). Diagnosis and management of head and neck cancer: a national clinical guideline; available at http://www.sign.ac.uk/pdf/sign90.pdf

4. Deary I.J., Wilson J.A., Carding P.N., MacKenzie K. (2003) VoiSS: A patient-derived Voice Symptom Scale. Journal of Psychosomatic Research. 54, 483-489.

5. Bowling A., Rowe G., Lambert N., Waddington M., Mahtani K.R., Kenten C. et al. (2012) The measurement of patients' expectations for health care: a review and psychometric testing of a measure of patients' expectations. Health Technology Assessment. 16, 30.

6. Jacobsen BH, Johnson A, Grywalski C, Silbergleit A, Jacobsen G, Benninger M, Newman CW. (1997) The voice handicap index (VHI): development and validation. Am J Speech Lang Pathol. 6, 66-70.

7. Rosen CA, Lee AS, Osbourne J, Zullo T, Murray T. (2004) Development and validation of the voice handicap index-10. Laryngoscope. 114, 1549-56.

8. Hogikyan ND, Sethuraman G. (1999) Validation of an instrument to measure voice-related quality of life (VRQOL). J Voice. 3, 557-569.

9. Robinson K., Gatehouse S., Browning G.G. (1996) Measuring patient benefit from otorhinolaryngological surgery and therapy. Ann Otol Rhinol Laryyngol. 105, 415-422.

10. Virk J.S., Awad Z., Singh A., Khalil S. (2014) Patients expectations in day surgery unit: Our experience. Clin Otolaryngol. 39, 379-384.

11. Awad Z., Virk J.S., Singh A. (2014) Patient expectations in outpatient care. Clin Otolaryngol. 39, 74-75. 
Table 1 - Number of responses to individual factors within PEQ

\begin{tabular}{|c|c|}
\hline Question & Number of responses \\
\hline 1. I want to know what is wrong with my voice & $342(75.2 \%)$ \\
\hline 2. I want to know what is wrong with my throat & $359(78.9 \%)$ \\
\hline 3. I want to know what is wrong/ I want a diagnosis & $345(75.8 \%)$ \\
\hline 4. I want to know if I have cancer & $226(49.7 \%)$ \\
\hline 5. I want to know if I have something seriously wrong & $288(63.3 \%)$ \\
\hline 6. I want to know why I have pain in my throat & $157(34.5 \%)$ \\
\hline 7. I want to know why I have problems with swallowing & $134(29.5 \%$ \\
\hline 8. I want to know why I have lots of phlegm or catarrh or mucous in my throat & $204(44.8 \%)$ \\
\hline 9. Tell me what I can do to make my throat problem better & $341(74.9 \%)$ \\
\hline 10. Tell me how I can improve my voice/ I want my voice improved & $252(55.4 \%)$ \\
\hline 11. I want to know what the ENT team can do to make me better & $310(68.1 \%)$ \\
\hline 12. I want my singing voice better & $109(24 \%)$ \\
\hline 13. GP/other doctor wanted me checked out & $364(80 \%)$ \\
\hline 14. None/ I don't know why I am here & $16(3.5 \%)$ \\
\hline
\end{tabular}


Table 2 - Mean age of patients per individual factors within PEQ

\begin{tabular}{|c|c|}
\hline Question & Mean age in years (SD) \\
\hline 1. I want to know what is wrong with my voice & $52(18.4)$ \\
\hline 2. I want to know what is wrong with my throat & $52(19)$ \\
\hline 3. I want to know what is wrong/ I want a diagnosis & $52.7(18.9)$ \\
\hline 4. I want to know if I have cancer & $55.7(18)$ \\
\hline 5. I want to know if I have something seriously wrong & $52.4(18.8)$ \\
\hline 6. I want to know why I have pain in my throat & $50.1(18)$ \\
\hline 7. I want to know why I have problems with swallowing & $53.8(18.1)$ \\
\hline 8. I want to know why I have lots of phlegm or catarrh or mucous in my throat & $55(19.2)$ \\
\hline 9. Tell me what I can do to make my throat problem better & $52.7(19)$ \\
\hline 10. Tell me how I can improve my voice/ I want my voice improved & $51.3(19.1)$ \\
\hline 11. I want to know what the ENT team can do to make me better & $52.5(19.1)$ \\
\hline 12. I want my singing voice better & $48.5(19.6)$ \\
\hline 13. GP/other doctor wanted me checked out & $53.3(18.5)$ \\
\hline 14. None/ I don't know why I am here & $58.4(20)$ \\
\hline
\end{tabular}




\section{Patient Expectation Questionnaire (PEQ)}

Date

We would like to know what you want from your visit to the clinic. Please tick all that apply:-

1. I want to know what is wrong with my voice

2. I want to know what is wrong with my throat

3. I want to know what is wrong/I want a diagnosis

4. I want to know if I have cancer

5. I want to know if I have something seriously wrong

6. I want to know why I have pain in my throat

[ ]

7. I want to know why I have problems with swallowing

8. I want to know why I have lots of phlegm or catarrh or mucous in my throat

9. Tell me what I can do to make my throat problem better

10. Tell me how I can improve my voice/ I want my voice improved

11. I want to know what the ENT team can do to make me better

12. I want my singing voice better

13. GP/other doctor wanted me checked out

14. None/ I don't know why I am here

15. Anything else

Thank you. Please hand back to a member of staff in clinic. 
Assessing and Caring for Patient Expectations in Laryngology (ACaPELa)

Date

We would like to know what you want from your visit to the clinic. Please rank all questions below that apply with 1-8 ( 1 being most important and 8 being least important - you do not need to rank all questions)

1. I want to know what is wrong with my voice

2. I want to know what is wrong with my throat

3. I want to know what is wrong/I want a diagnosis

4. I want to know if I have cancer

5. I want to know if I have something seriously wrong

6. Tell me what I can do to make my throat problem better

7. Tell me how I can improve my voice/ I want my voice improved

8. I want to know what the ENT team can do to make me better

Thank you. Please hand back to a member of staff in clinic. 